\title{
Suicidal Behaviors in a Nationally Representative Sample of School-Going Adolescents Aged 12-17 Years in Eswatini
}

\author{
Emmanuel Nii-Boye Quarshie ${ }^{1,2}$ (D) Prince Atorkey ${ }^{3,4}$. \\ Karla Patricia Valdés García ${ }^{5}$. Samuel Afotey Lomotey ${ }^{6}$. \\ Pascal Landindome Navelle ${ }^{7}$
}

Accepted: 16 July 2021 /Published online: 2 August 2021

(c) The Author(s) 2021

\begin{abstract}
The burgeoning body of evidence suggests that the aggregated 12-month prevalence estimates of suicidal behaviors (ideation, planning, and attempt) are relatively higher among in-school adolescents in Africa, although country-specific data on the phenomena are still inadequate from the continent. We sought to estimate the 12-month prevalence and identify some of the correlates of suicide behaviors among schoolgoing adolescents in Eswatini. We analyzed the nationally representative data from the 2013 Eswatini World Health Organization Global School-based Student Health Survey, using univariate, bivariate, and multivariate statistical approaches. Of the 2,513 analytical samples, $17.0 \%$ (95\% confidence interval [CI]: 15.4-18.4\%) reported suicidal ideation, $21.0 \%$ (95\% CI: $19.3-22.6 \%)$ made a suicide plan, and $15.5 \%$ (95\% CI: 14.1-16.9\%) attempted suicide during the previous 12 months. The final adjusted logistic models indicated health risk behavior (i.e., marijuana use), adverse interpersonal factors within the family (e.g., intrusion of privacy by parents), and school contextual factors (e.g., bullying victimization, physical fights) to be associated with increased odds of suicidal behaviors among females. However, predominantly, school-related interpersonal factors (e.g., bullying victimization, physical fights) showed strong associations with increased odds of suicidal behaviors among males. Generally, parental monitoring, parental understanding, and social support at school were associated with reduced odds of suicidal behaviors. The multi-level nature of our findings underscores the need for multi-contextual and multi-sectoral intervention and prevention programs and policy approaches targeted at mitigating the onset of suicidal ideation and possible transition to suicidal planning, attempt, and potential death by suicide in this young population.
\end{abstract}

Keywords Adolescents · Attempted suicide $\cdot$ Eswatini $\cdot$ Ideation · Suicidal behavior · Suicide

Emmanuel Nii-Boye Quarshie

e.n.quarshie@leeds.ac.uk; enquarshie@gmail.com

Extended author information available on the last page of the article 


\section{Introduction}

Suicidal behavior is used to mean "a range of behaviors that include thinking about suicide (or ideation), planning for suicide, attempting suicide and suicide itself" (WHO, 2014, p. 12). Suicidal ideation, planning, and (history of) attempted suicide are critical risks for suicidal death among all age groups within the general population (Klonsky et al., 2016; Turecki et al., 2019; WHO, 2014). This evidence underscores suicidal ideation, planning, and attempt as important suicide research foci, towards informing intervention and prevention efforts against deaths by suicide (Hawton et al., 2012; Nock, 2012; Turecki et al., 2019; Zalsman et al., 2016).

Recent evidence suggests that globally, approximately, 4 out of 100,000 young persons aged 10-19 years take their lives annually (Glenn et al., 2020), ranking suicide as the second leading cause of death among young persons aged 15-19 years (WHO, 2019). Most of what is known about suicidal behaviors among adolescents in low- and middle-income countries (LAMICs) is based on research from high-income countries (Aggarwal et al., 2017; Biswas et al., 2020; Glenn et al., 2020; Lim et al., 2019; Quarshie et al., 2020c). Globally, varying pooled 12-month prevalence estimates of suicidal behaviors have been reported among adolescents (suicide ideation $=14.2 \%$ (95\% confidence interval $[\mathrm{CI}]=11.6-17.3)$, suicidal planning $=7.5 \%(\mathrm{CI}=4.5-12.1)$, and suicide attempt $=4.5 \%(95 \% \mathrm{CI}=3.4-5.9)]$ (Lim et al., 2019). Relative to other regions of the world, the burgeoning body of systematic reviews and meta-analyses of school-based health survey findings across LAMICs suggests that the aggregated 12-month prevalence estimates of suicidal behaviors are higher among inschool adolescents in Africa (suicidal ideation $=21 \%(95 \% \mathrm{CI}=20.1-21.0)$, suicide planning $=23.7 \%(95 \% \mathrm{CI}=19.1-28.3)$, and suicide attempt $=16.3 \%(95 \%$ $\mathrm{CI}=8.4-29 \%)$ (Biswas et al., 2020; Li et al., 2020; Liu et al., 2018; Uddin et al., 2019).

A recent systematic review pooling evidence on self-harm among both inschool and "out-of-school" adolescents in sub-Saharan Africa has reported a 12-month median prevalence estimate of $16.5 \%$ (interquartile range $[\mathrm{IQR}]=10.9-24.0 \%)$ from Southern sub-Saharan Africa — where Eswatini is located (Quarshie et al., 2020c). Whereas evidence from high-income countries indicates that both prevalence estimates of suicidal ideation, planning, and attempt are higher among female adolescents than males (Hawton et al., 2012; Muehlenkamp et al., 2012; Swannell et al., 2014), the research findings from sub-Saharan Africa have shown mixed gender patterns (Quarshie et al., 2020c). Some studies have found comparable estimates between males and females (Liu et al., 2018; Quarshie \& Andoh-Arthur, 2020), other studies have found higher estimates among males (Peltzer \& Pengpid, 2017; Shaikh et al., 2016), while some studies have reported higher estimates among females (Asante et al., 2017; McKinnon et al., 2016). Even though these gender differences may be attributable to variations in the definitions and measurements of key outcome variables, methods, contexts, sampling, and sample characteristics of available studies, the 
most critical reason is the general paucity of studies on suicidal behaviors among young people in sub-Saharan African countries. More studies (involving the use of robust and carefully designed approaches) are needed to help map out the gender differences (if any exists) across the subregion (Quarshie et al., 2020c).

Emerging evidence from LAMICs indicates that multiple factors are associated with suicidal behaviors among school-attending adolescents in sub-Saharan Africa (Aggarwal et al., 2017; Biswas et al., 2020; Campisi et al., 2020; Li et al., 2020; Liu et al., 2018; McKinnon et al., 2016; Quarshie et al., 2020c). The factors are also multi-layered, existing at the personal/individual level (e.g., gender, hopelessness, low self-worth, alcohol and drug misuse, psychiatric symptoms/disorders including depression and anxiety, chronic illness such as HIV/AIDS), and various socio-ecological contexts within which adolescents live: family context (e.g., parental divorce, conflict with parents, conflict between parents, family history of suicide or attempted suicide, physical abuse, familial poverty, orphanhood, parental understanding, and support), school environment (bullying victimization, poor school climate, lack of peer support, peer suicide or attempted suicide, romantic relationships problems and breakups, poor academic performance, and truancy), and the broader community context (sexual abuse, food insecurity, war/community violence, child marriage, and poverty) (Quarshie et al., 2020c).

Considering that studies on behavioral and emotional outcomes in adolescents have shown that females score higher on internalizing problems than males (Angold et al., 2002; Rescorla et al., 2007), it is important for research to examine the genderspecific factors associated with suicidal behaviors among adolescents, in order to inform effective universal and targeted intervention and prevention programs. However, there is still a lack of comprehensive studies from Africa that asses the genderspecific factors associated with suicidal behaviors among school-going adolescents; generally, country-specific evidence on adolescent suicidal behaviors (ideation, planning, and attempt) is inadequate from Africa (Aggarwal et al., 2017; Mortier et al., 2018; Quarshie et al., 2020c).

The initiative of the World Health Organization Global School-based Student Health Survey (WHO-GSHS) is worth noting as a critical contribution to understanding adolescent suicidal behaviors across LAMICs, providing essential data in different countries, including those in Africa (Campisi et al., 2020; Quarshie et al., 2020c; Uddin et al., 2019). The WHO-GSHS is a surveillance project designed collaboratively by the WHO, UNAIDS, UNESCO, UNICEF, and the US Centers for Disease Control and Prevention (CDC) (WHO, 2020). The WHO-GSHS began in 2003 to support participating countries to assess the behavioral risks and protective factors among young people aged 13-17 years. Currently, 18 countries (including Eswatini) from sub-Saharan Africa participate in the WHO-GSHS (WHO, 2020). Data from the WHO-GSHS are made publicly accessible and available to inform national and cross-country analyses and peer-reviewed publications, to inform intervention and prevention programs in the respective countries and regions. However, to date, we are not aware of any comprehensive peer-reviewed publication of the WHO-GSHS latest data from Eswatini (collected in 2013) on suicidal behaviors i.e., prevalence and correlates of suicidal planning and attempt, although countryspecific publications on the phenomena based on the WHO-GSHS data from other 
parts of sub-Saharan Africa are available (Asante et al., 2017; Peltzer \& Pengpid, 2017; Pengpid \& Peltzer, 2020b; Quarshie et al., 2020a; Randall et al., 2014; Rudatsikira et al., 2007; Shaikh et al., 2016; Shayo \& Lawala, 2019; Wilson et al., 2012). A comprehensive analysis of the WHO-GSHS Eswatini data will, among other useful purposes, allow for comparison with the published WHO-GSHS data on suicidal behaviors from other countries in (sub-Saharan) Africa.

Thus far, while only two recent country-specific studies have drawn on separate household survey data to report the prevalence and associated factors of suicidal behaviors among adolescents in Eswatini (Berhane et al., 2020; Pengpid \& Peltzer, 2020a), only one country-specific study has analyzed the 2013 Eswatini WHOGSHS dataset on suicide ideation (12-month prevalence estimates and associated factors) (Almansour \& Siziya, 2017). Notably, several recent cross-national secondary analyses of the WHO-GSHS data from Africa have included the dataset from Eswatini, without reporting the country-specific correlates of suicidal ideation, planning, and attempt (Campisi et al., 2020; Li et al., 2020; Liu et al., 2018). Therefore, the current study seeks to analyze the 2013 Eswatini WHO-GSHS data on suicidal behaviors to address the following research questions:

i) What are the 12-month prevalence estimates of suicidal ideation, planning, and attempt among school-going adolescents in Eswatini, and do the estimates differ for males and females?

ii) What are the personal/lifestyle-, family-, school-, and interpersonal relationship-related factors associated with suicidal behaviors among school-going adolescents in Eswatini, and do these factors differ for males and females? Considering the remit of the data analyzed in the current study, the analysis considers suicidal behaviors to be composed of suicidal ideation, planning, and attempt.

\section{Methods}

The study was reported according to Strengthening the Reporting of Observational Studies in Epidemiology (STROBE) criteria (Vandenbroucke et al., 2007).

\section{Study design}

As applied in all participating countries, the 2013 Eswatini WHO-GSHS used a cross-sectional survey design involving a structured self-report questionnaire (WHO, 2020).

\section{Study context}

Eswatini is a lower middle-income country in Southern sub-Saharan Africa with a general population of 1,093,238 people, of which about 37\% are aged 15-34 years (Government of the Kingdom of Eswatini \& UNFPA, 2019). Eswatini is a medium human development index country (HDI rank=0.611) with a life expectancy at 
birth of 60.2 years (UNDP, 2020). The country's population is youthful, as more than 120,000 are aged $15-19$ years; expected years of schooling is $11.8($ mean $=6.9)$ (Government of the Kingdom of Eswatini \& UNFPA, 2019; UNDP, 2020). Adolescents and young people (particularly, those aged 15-24 years) in the country face many challenges, including alcohol and substance use problems, early marriage, orphanhood, teenage pregnancy, maternal mortality, a relatively high incidence of HIV, and dropping out of school (Government of the Kingdom of Eswatini \& UNFPA, 2019; Ministry of Education and Training \& UNICEF, 2018; Ministry of Sports Culture \& Youth Affairs, 2015). For example, in 2018, the estimated net primary school enrollment was $95 \%$, whereas secondary school net enrollment rate was $46.3 \%$ - indicating a high attrition rate in the transition from primary school to secondary school, resulting in low educational attainment (Government of the Kingdom of Eswatini \& UNFPA, 2019).

\section{Data collection}

Data for this study was obtained from the 2013 Eswatini WHO-GSHS. The GSHS data are collected from a nationally representative sample of secondary school students after ethical approval and the relevant authorities grant other permissions. Students who voluntarily consent to complete the survey record their answers on a computer-scannable form distributed by trained staff during one standard class period. No individually identifiable information is collected. Additional methodological details about the 2013 Eswatini GSHS have been reported by an earlier publication (Almansour \& Siziya, 2017). As shown in Fig. 1, we applied a set of inclusion and exclusion criteria to obtain an analytical sample of adolescents aged 12-17 years, the typical age range of secondary school students in Eswatini.

Overall, 3,680 students responded to the survey, but our final analytical sample included 2,513 after the data selection criteria were applied (see Fig. 1). Of the 2,513 students aged between 12 and 17 years $($ mean $=15.4$ years, standard deviation $=1.4$ ) who provided complete data, 1,083 (43.1\%) were males and 1,430 (56.9\%) were females (Fig. 1).

The survey procedure followed the Eswatini Ministry of Education and Training and the ethical requirements for recruiting students for research. The Institutional Review Board of the Country Office of WHO in Eswatini, and the Eswatini Ministry of Education and Training, approved the survey (WHO, 2020).

\section{Measures}

Demographic variables Gender (male or female) and age were demographic variables included in this study.

Exposure variables Drawing on previous publications based on the WHO-GSHS data (Almansour \& Siziya, 2017; Asante et al., 2017; Campisi et al., 2020; Pengpid \& Peltzer, 2020b; Shayo \& Lawala, 2019), the following variables were considered potential correlates of the outcome variables: mental health and lifestyle (loneliness, 


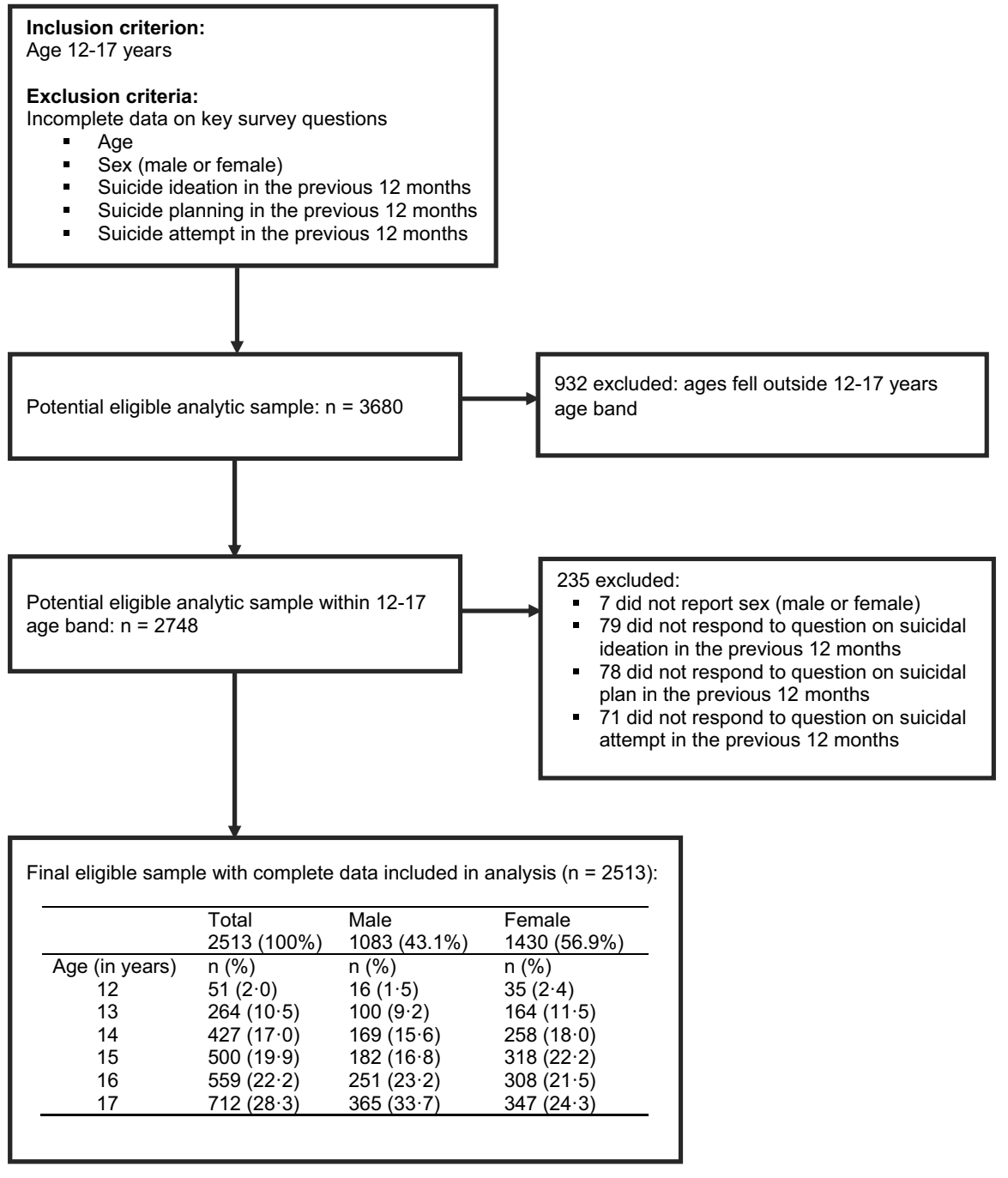

Fig. 1 Flow diagram of analytic sample selection process and criteria

anxiety, and marijuana use); family-level factors (i.e., parental monitoring, parental understanding, parental supervision, and food deprivation); school-level factors (i.e., truancy, bullying victimization, and social support at school); and interpersonallevel factors (i.e., being sexually active, number of close friends, and physical fight).

Outcome variables This study's outcome variables were suicide ideation, suicide plan, and suicide attempt in the past 12 months. Each of these variables was measured using a single item on the questionnaire: suicide ideation ("During the past 12 months, did you seriously consider attempting suicide?"), suicide plan ("During the past 12 months, did you make a plan about how you would attempt 
suicide?"), suicide attempt ("During the past 12 months, how many times did you actually attempt suicide?"). See e-Table 1 in Supplementary Material for all the variables included in this study, the questions used in assessing each variable, and how responses were coded for statistical analysis.

\section{Statistical analysis}

Three statistical analyses were performed using SPSS (version 27 for windows):

1) Univariate analysis (i.e., percentages and frequencies) was performed to determine the 12-month prevalence estimates of suicidal ideation, planning, and attempt;

2) Bivariate analysis using chi-square $\left(\chi^{2}\right)$ to determine the relationship between the exposure variables and each of the outcome variables; and

3) Multivariate models, one each for the outcome variables involving logistic regression, were used to determine the demographic factors and exposure variables associated with the outcome variables (suicidal ideation, planning, and attempt).

To avoid overfitting the models, the demographic and exposure variables were entered into the logistic regression models regardless of their significant bivariate associations with the outcome variables (Babyak, 2004; Sun et al., 1996). The two demographic variables were included as covariates in all three multivariate models. The logistic regression modeling results were reported as adjusted odd ratios (AOR) with their associated $95 \%$ confidence intervals (95\% CI). Guided by the research questions of the study, the bivariate and multivariate analyses were stratified by gender. Missing responses were excluded from the analysis; e-Table 1 (in Supplementary Material) shows the proportion of missing data related to each variable. The statistical significance threshold was set at $p<0.05$.

Data for study is publicly accessible and available on the WHO website: https:// www.who.int/ncds/surveillance/gshs/swaziland/en/.

\section{Results}

\section{Prevalence of suicidal ideation, planning, and attempt}

Overall, $17.0 \%(95 \% \mathrm{CI}=15.4-18.4 \%)$ of the total analytic sample reported suicidal ideation during the previous 12 months, representing $15.0 \%$ (95\% CI=12.9-17.2\%) among males and $18.3 \%(95 \% \mathrm{CI}=16.3-20.4 \%)$ among females. This prevalence estimate was significantly higher among females than males $\left(15.0 \%\right.$ vs. $18.3 \% ; \chi^{2}$ (1) $=4.9, p=0.026$ ) (see e-Table 2 in Supplementary Material).

The overall 12-month prevalence estimate of suicidal planning was $21.0 \%$ (95\% CI $=19.3-22.6 \%)$. This suicidal planning estimates were comparable between males $(20.0 \%$ [95\% CI $=17.7-22.5 \%])$ and females $(21.6 \%$ [95\% 
$\mathrm{CI}=19.5-23.8 \%])$ : the gender difference in the estimates did not reach the desired statistical significance threshold $\left(20.0 \%\right.$ vs. $\left.21.6 \% ; \chi_{(1)}^{2}=0.9, p=0.338\right)$ (see e-Table 3 in Supplementary Material).

Also, the overall 12-month prevalence estimate of suicidal attempt $(15.5 \%$ [95\% CI 14.1-16.9\%]) was similar between males $(15.0 \%$ [95\% CI $=12.9-17.2 \%])$ and females $(15.9 \%$ [(95\% CI $=14.1-17.9 \%])$. Again, the gender difference in the estimates did not reach the desired threshold of statistical significance $(15.0 \% \mathrm{vs}$. $15.9 \% ; \chi_{(1)}^{2}=0.5, p=0.499$ ) (see e-Table 4 in Supplementary Material).

Overall, the bivariate analysis showed many associations with suicidal ideation, planning, and attempt (see e-Tables 2, 3, \& 4, in Supplementary Material). Predominantly, the factors with statistically significant bivariate associations with suicidal ideation, planning, and attempt were found within the school context (bullying victimization, truancy, and social support at school), interpersonal relationships (engaging in physical fights, being sexually active), and those that were related to mental health and lifestyle factors (loneliness, anxiety, and marijuana use).

\section{Factors associated with suicidal ideation, planning, and attempt}

\section{Suicidal ideation}

Overall, seven factors were associated with increased odds of suicidal ideation among the participants: marijuana use $(\mathrm{AOR}=2.04,95 \% \mathrm{CI}=1.13$, $3.67 ; p=0.018$ ), truancy ( $\mathrm{AOR}=1.83,95 \% \mathrm{CI}=1.30,2.56 ; p<0.001)$, bullying victimization $(\mathrm{AOR}=1.75,95 \% \mathrm{CI}=1.34,2.28 ; p<0.001)$, being sexually active $(\mathrm{AOR}=1.62,95 \% \mathrm{CI}=1.15,2.27 ; p=0.006)$, anxiety $(\mathrm{AOR}=1.56$, $95 \% \mathrm{CI}=1.18,2.06 ; p=0.002)$, loneliness $(\mathrm{AOR}=1.39,95 \% \mathrm{CI}=1.03,1.88$; $p=0.030)$, and female gender $(\mathrm{AOR}=0.69,95 \% \mathrm{CI}=0.52,0.90 ; p=0.006)$. However, parental monitoring $(\mathrm{AOR}=0.74,95 \% \mathrm{CI}=0.55,0.99 ; p=0.043)$ and parental understanding $(\mathrm{AOR}=0.71,95 \% \mathrm{CI}=0.52,0.97 ; p=0.030)$ were associated with reduced odds of suicidal ideation - see Table 1.

In the subgroup analysis, truancy $(\mathrm{AOR}=2.38,95 \% \mathrm{CI}=1.45,3.91$; $p=0.001)$ and having engaged in physical fights $(\mathrm{AOR}=1.65,95 \% \mathrm{CI}=1.05$, $2.60 ; p=0.031)$ were associated with increased odds of suicidal ideation among males, while marijuana use $(\mathrm{AOR}=3.13,95 \% \mathrm{CI}=1.06,9.26 ; p=0.039)$, being sexually active $(\mathrm{AOR}=2.40,95 \% \mathrm{CI}=1.49,3.87 ; p<0.001)$, and anxiety $(\mathrm{AOR}=1.77,95 \% \mathrm{CI}=1.22,2.57 ; p=0.003)$ emerged as significant associates of increased odds of suicidal ideation among females. Notably, however, bullying victimization was the only factor commonly associated with increased odds of suicidal ideation between male $(\mathrm{AOR}=1.91,95 \% \mathrm{CI}=1.24,2.96$; $p=0.004)$ and female $(\mathrm{AOR}=1.65,95 \% \mathrm{CI}=1.17,2.32 ; p=0.004)$ participants (see Table 1); both males and females who reported bullying victimization were about two times likely to experience suicidal ideation, compared to their counterparts who were not bullied. 


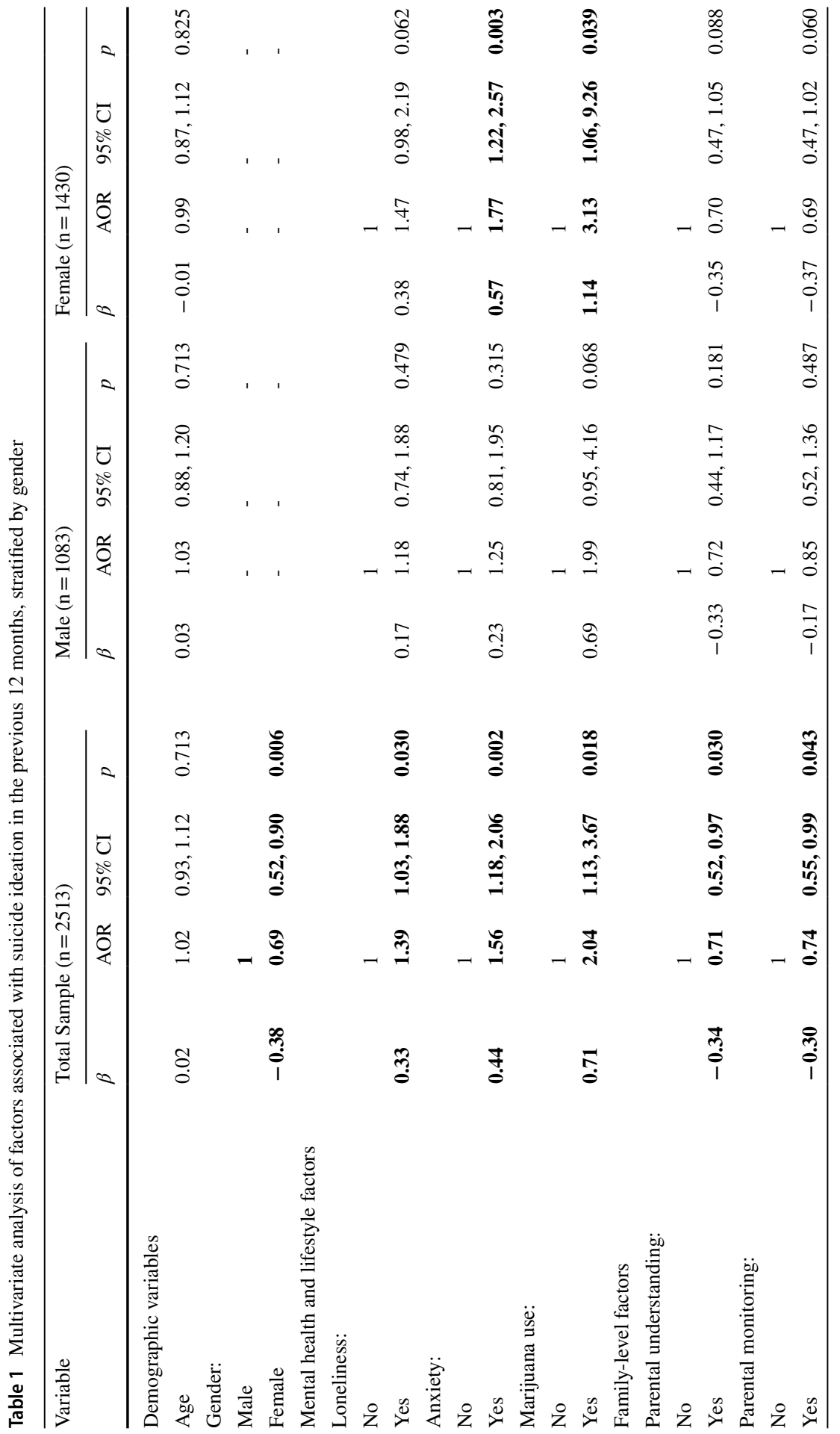




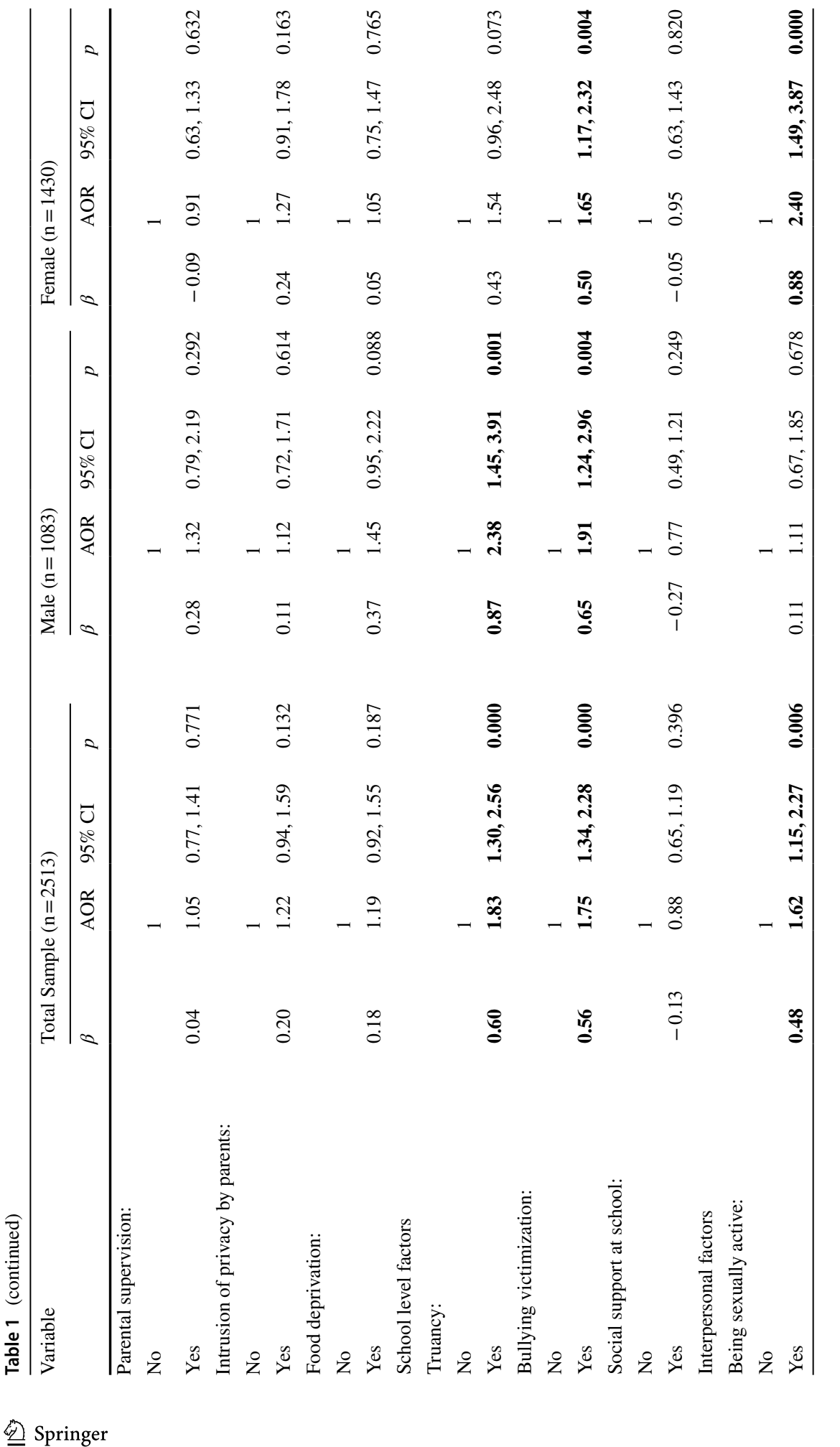




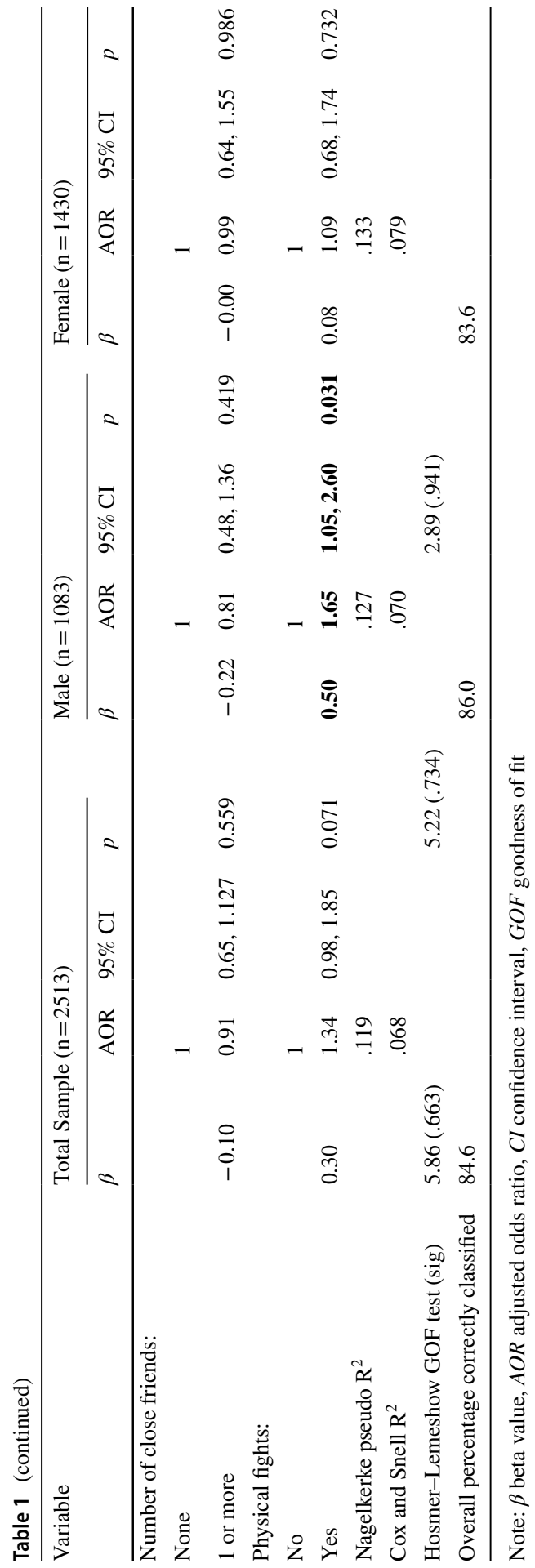




\section{Suicidal planning}

Table 2 shows that overall, eight factors were associated with increased odds of suicidal planning among the participants: marijuana use $(\mathrm{AOR}=2.53,95 \% \mathrm{CI}=1.45$, $4.42 ; p=0.001$ ), physical fights ( $\mathrm{AOR}=1.57,95 \% \mathrm{CI}=1.17,2.11 ; p=0.002)$, truancy (AOR $=1.46,95 \% \mathrm{CI}=1.05,2.02 ; p=0.024)$, being sexually active (AOR $=1.43,95 \%$ $\mathrm{CI}=1.03,1.98 ; p=0.030)$, intrusion of privacy by parents $(\mathrm{AOR}=1.41,95 \% \mathrm{CI}=1.11$, $1.79 ; p=0.005$ ), food deprivation ( $\mathrm{AOR}=1.33,95 \% \mathrm{CI}=1.05,1.69 ; p=0.018$ ), bullying victimization $(\mathrm{AOR}=1.29,95 \% \mathrm{CI}=1.00,1.69 ; p=0.047)$, and female gender $(\mathrm{AOR}=0.71,95 \% \mathrm{CI}=0.55,0.91 ; p=0.006)$. Only social support at school (AOR $=0.75,95 \% \mathrm{CI}=0.57,0.98 ; p=0.033$ ) showed a significant association with reduced odds of suicidal planning.

Among the male subgroup, food deprivation $(\mathrm{AOR}=1.78,95 \% \mathrm{CI}=1.21,2.62$; $p=0.004)$ and physical fights $(\mathrm{AOR}=1.75,95 \% \mathrm{CI}=1.13,2.72 ; p=0.012)$ were associated with increased odds of suicidal planning, whereas social support at school $(\mathrm{AOR}=0.64,95 \% \mathrm{CI}=0.43,0.96 ; p=0.031)$ emerged as the only factor associated with reduced odds of suicidal planning in males. Among the female participants, marijuana use $(\mathrm{AOR}=5.50,95 \% \mathrm{CI}=1.88,16.08 ; p=0.002)$ and intrusion of privacy by parents $(\mathrm{AOR}=1.41,95 \% \mathrm{CI}=1.04,1.92 ; p=0.028)$ showed significant association with increased odds of suicidal planning. As indicated in Table 2, no factor emerged in the subgroup analysis to be commonly associated with suicidal planning between males and females.

\section{Suicidal attempt}

Taken together, Table 3 shows that marijuana use $(\mathrm{AOR}=2.89,95 \% \mathrm{CI}=1.61$, 5.16; $p<0.001$ ), physical fights ( $\mathrm{AOR}=1.83,95 \% \mathrm{CI}=1.33,2.51 ; p<0.001)$, bullying victimization ( $\mathrm{AOR}=1.47,95 \% \mathrm{CI}=1.47,1.11 ; p=0.007)$, food deprivation $(\mathrm{AOR}=1.36,95 \% \mathrm{CI}=1.04,1.77 ; p=0.026)$, and female gender $(\mathrm{AOR}=0.71,95 \%$ $\mathrm{CI}=0.54,0.93 ; p=0.014)$ were associated with increased odds of suicidal attempt among the participants.

The subgroup analysis showed that while bullying victimization $(\mathrm{AOR}=1.72,95 \%$ $\mathrm{CI}=1.10,2.70 ; p=0.018)$ was uniquely associated with increased odds of suicidal ideation among males, physical fights $(\mathrm{AOR}=2.29,95 \% \mathrm{CI}=1.49,3.51 ; p<0.001)$ emerged as a significant associate of increased odds of suicidal attempt in females. The model (Table 3) showed marijuana use as the only factor commonly associated with increased odds of suicidal attempts in both male $(\mathrm{AOR}=3.0,95 \% \mathrm{CI}=1.47,6.11$; $p=0.002)$ and female ( $\mathrm{AOR}=2.94,95 \% \mathrm{CI}=1.01,8.54 ; p=0.048)$ participants. Specifically, both males and females who reported marijuana use were about three times more likely to report attempted suicide during the previous 12 months than their counterparts who reported no marijuana use. 


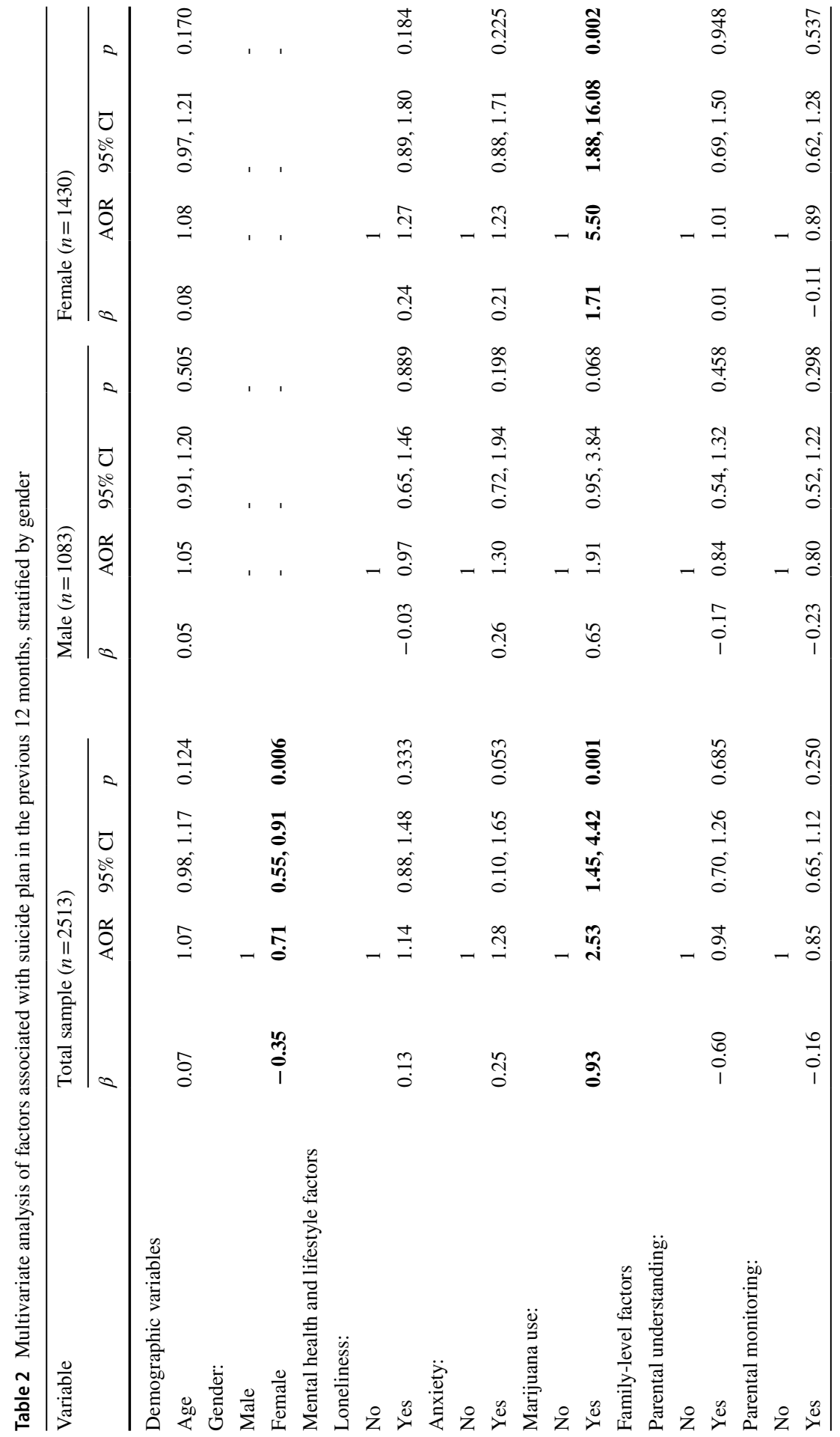




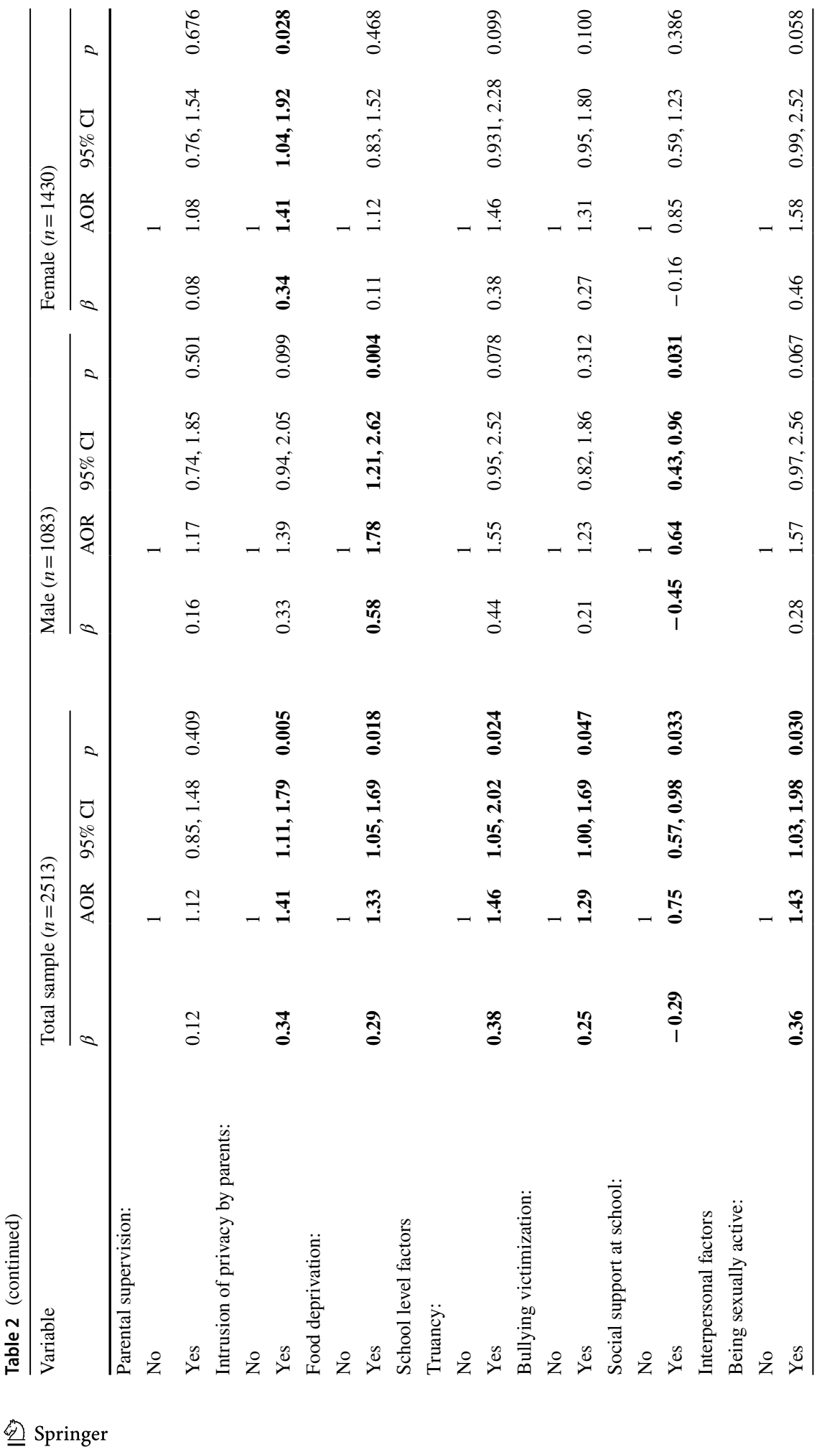




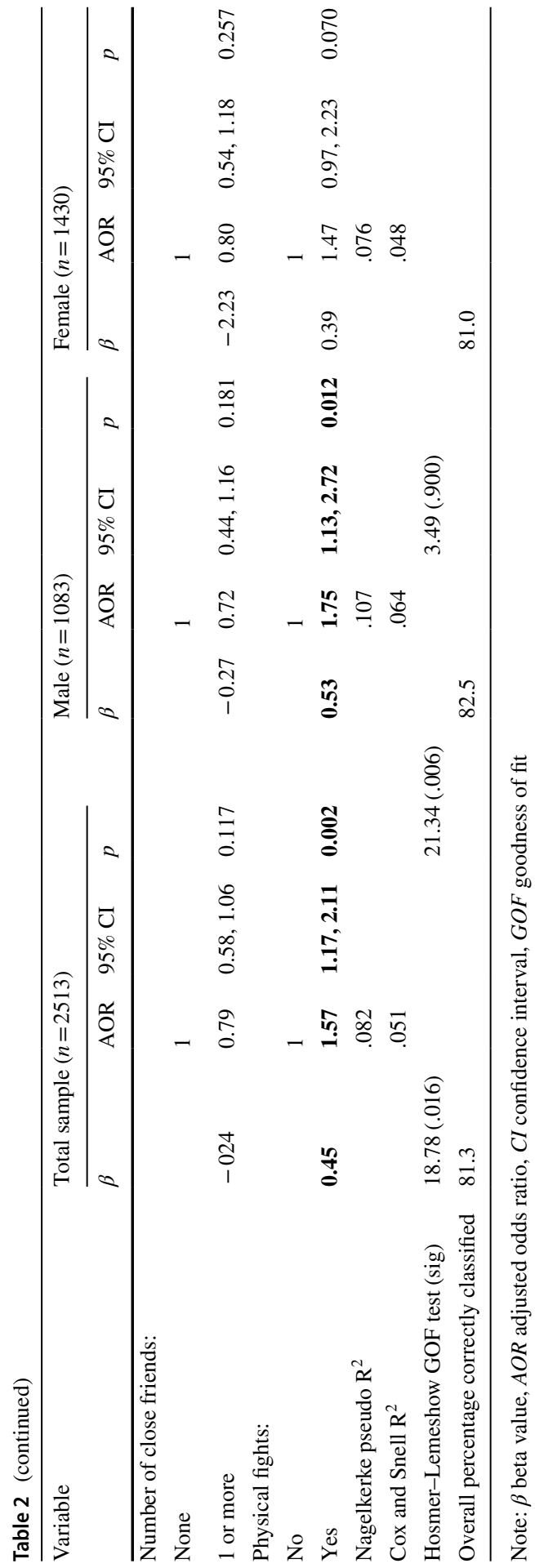




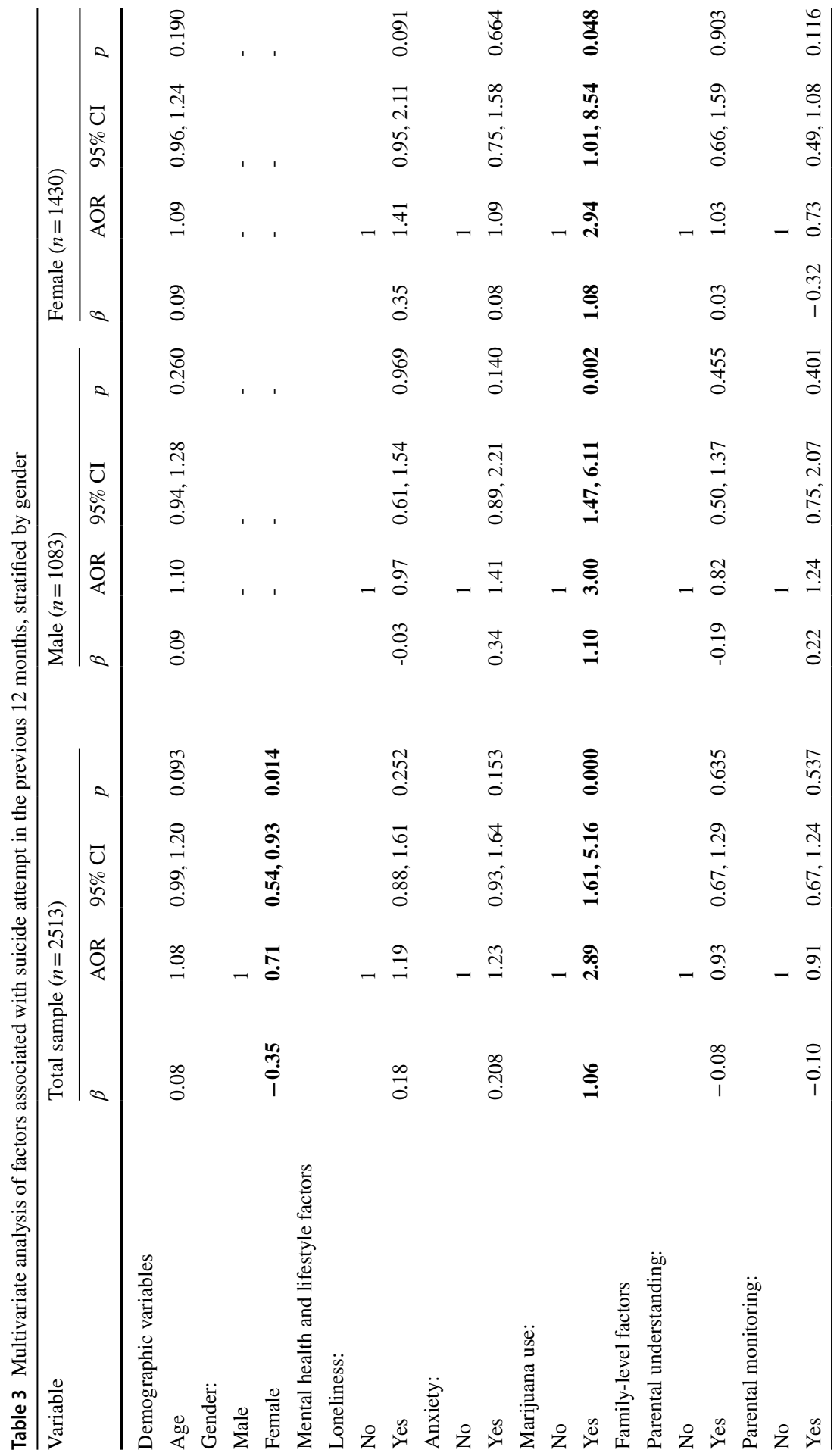




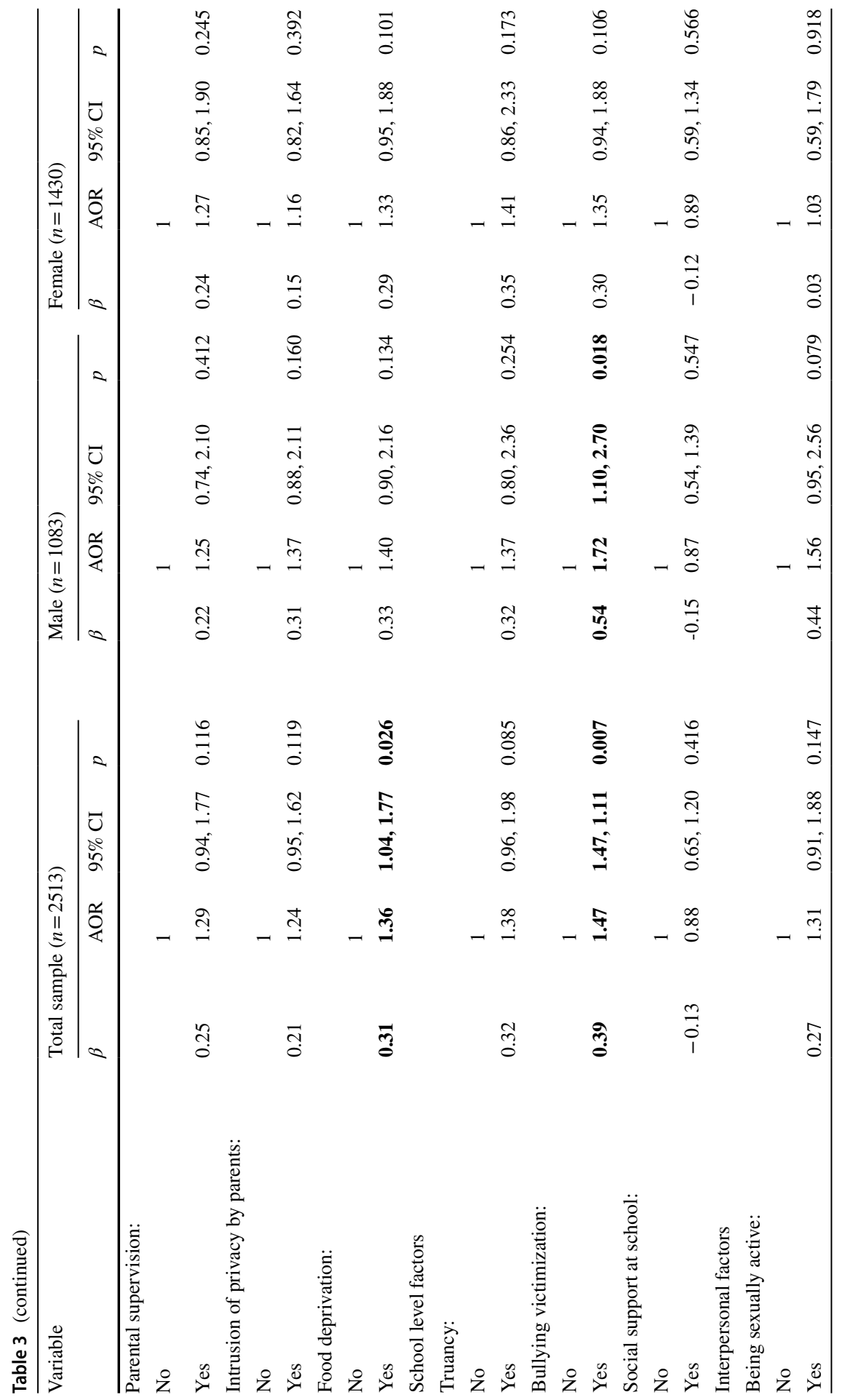




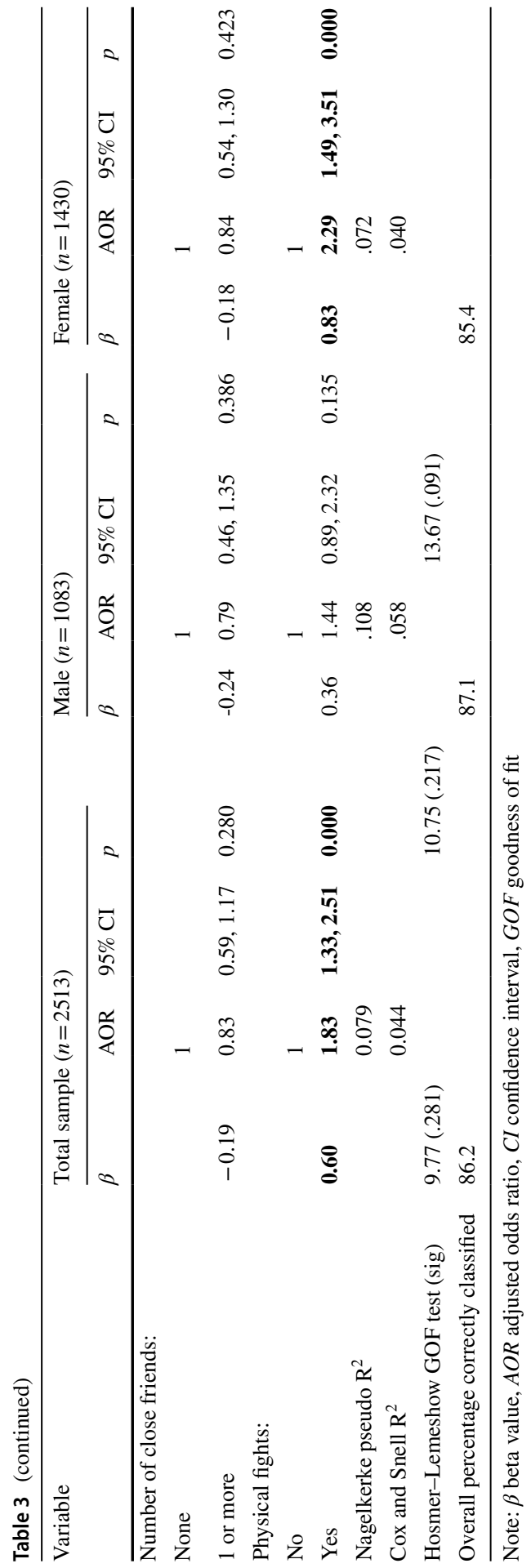




\section{Discussion}

In this secondary analysis of the 2013 Eswatini WHO-GSHS data, we have found that approximately 1.7 out of 10 participants reported suicidal ideation, 1 out of 5 reported suicidal planning, and about 1.5 out of 10 participants attempted suicide during the previous 12 months. The prevalence estimate of suicidal ideation was higher among females than males, while the estimates of suicidal planning and attempt were comparable between females and males. Multivariate statistical modeling of the data showed the female gender to be associated with increased odds of suicidal ideation, planning, and attempt during the previous 12 months. Among females, the multivariable statistical modeling found health risk behavior (i.e., marijuana use), adverse interpersonal factors within the family (e.g., intrusion of privacy by parents), and school contextual factors (e.g., bullying victimization, physical fights) to be associated with increased odds of suicidal behaviors. However, predominantly, school-related interpersonal factors (e.g., bullying victimization, social support at school, physical fights) showed strong associations with suicidal behaviors among males.

\section{Prevalence of suicidal behaviors}

The prevalence estimates observed in the current study are relatively higher than the known recent global estimates of suicidal behaviors among school-going adolescents (Lim et al., 2019); however, continentally and regionally, the reported 12-month prevalence estimates in the current study are comparable to the pooled estimates of recent studies from Africa and sub-Saharan Africa (Biswas et al., 2020; Campisi et al., 2020; Li et al., 2020; Liu et al., 2018; Uddin et al., 2019). More interestingly, within the specific context of sub-Saharan Africa, the estimate of suicide attempt reported in the current study $(15.5 \%$ [95\% CI 14.1-16.9\%]) is comparable to the pooled 12-month median prevalence estimate of $16.5 \%(\mathrm{IQR}=10.9-24.0 \%)$ observed recently from the Southern sub-Saharan African region (where Eswatini is located) (Quarshie et al., 2020c), but relatively lower than the estimate from Western sub-Saharan Africa (median $=24.3 \%$ [IQR $=16.9-27.9 \%]$ ) (Quarshie et al., 2020c). The reported estimate of suicidal ideation in the current study $(17.0 \%$ [95\% $\mathrm{CI}=15.4-18.4 \%])$ is strikingly similar to what has been reported (17.0\%) recently among in-school adolescents in Eswatini, drawing from the same 2013 Eswatini WHO-GSHS dataset (Almansour \& Siziya, 2017). Similarly, the estimates of suicidal planning and attempt in the current study are comparable to recently reported estimates based on the 2013 Eswatini WHO-GSHS dataset (Campisi et al., 2020; Li et al., 2020; Liu et al., 2018).

However, the prevalence estimates by the current study are higher relative to recent estimates from household and community-based studies involving adolescents in Eswatini — which have reported suicidal behavior to be $9.0 \%$ (Pengpid \& Peltzer, 2020a), 7.1\% in respect of suicidal ideation, and $5.3 \%$ in respect of suicidal attempt (Berhane et al., 2020). While the differences in the prevalence estimates 
between the school-based and community-based studies could be due to differences in sample characteristics and measures used, the differences could also be attributed to the possibility that the school environment in Eswatini may be presenting adolescents with adverse challenges and circumstances that potentially contribute to the onset of suicidal tendencies among secondary school students (Harber, 2017; Quarshie et al., 2020c).

Furthermore, the prevalence estimates of the current study are consistent with estimates from other Southern sub-Saharan African countries, including Namibia (Peltzer \& Pengpid, 2017), South Africa (James et al., 2017; Shilubane et al., 2013), and Mozambique (Pengpid \& Peltzer, 2020b; Seidu et al., 2020), which suggest that comparatively more school-attending female adolescents than males report suicidal behaviors. As observed by a recent systematic review on self-harm and suicidal behaviors among young people in sub-Saharan Africa (Quarshie et al., 2020c), it stands to suggest that the prevalence estimates reported in the current study could be pointing to the possibility that the precipitating factors of suicidal behaviors among school-going adolescents in Southern sub-Saharan African countries may be similar rather than varied.

\section{Factors associated with suicidal behaviors}

The multivariable statistical modeling results align with the socio-ecological framework for the understanding of suicidal behavior among adolescents (Centers for Disease Control \& Prevention, 2019; Perkins \& Hartless, 2002; Pfledderer et al., 2019; Shagle \& Barber, 1995; WHO, 2014). The analysis showed that the factors associated with suicidal ideation, planning, and attempt exist at multiple levels of the socio-ecological context of adolescents: individual-level factors (e.g., female gender, anxiety, loneliness, marijuana use), family environment factors (e.g., intrusion of privacy by parents, food deprivation, parental understanding), school-related factors (e.g., social support at school, bullying), and interpersonal factors (e.g., physical fights, being sexually active) are associated with suicidal behavior among school-going adolescents in Eswatini.

Specifically, the current study has shown the female gender (compared to male) to have a strong association with the odds of suicidal ideation, planning, and attempt. This evidence is consistent with previous findings from other countries in sub-Saharan Africa, including Malawi (Shaikh et al., 2016), Mozambique (Pengpid \& Peltzer, 2020b; Seidu et al., 2020), Ghana (Asante et al., 2017), South Africa (Shilubane et al., 2013), and Tanzania (Shayo \& Lawala, 2019). While this finding is worrying, it is not entirely surprising, considering the numerous telling psychosocial challenges that girls and women (relative to boys and men) experience in LAMICs generally, and specifically in sub-Saharan Africa (Petroni et al., 2015). In other words, the evidence that female adolescents - relative to males — are at elevated odds of suicidal behavior could be pointing to the high vulnerability of girls and women to rigid and unsupportive cultural norms, social and educational exclusion, discrimination, and abuse of various forms in sub-Saharan African countries and other resource-poor contexts (Kabiru, 2015; Kabiru et al., 2013). The implication is 
that clinical mental health support models and intervention should be supplemented with social and family interventions.

In terms of health risk behaviors associated with increased odds of suicidal behavior, marijuana use showed a strong association with suicidal ideation, planning, and attempt among females but was associated with only suicide attempts among males. On the one hand, this evidence is surprising, given that more male adolescents than females misuse marijuana; on the other hand, this evidence is to be expected considering the country-wide evidence that many school-going young people are involved in the production, transportation, and sale of marijuana in Eswatini (Ministry of Sports Culture \& Youth Affairs, 2015; Mkhatshwa \& Malambe, 2019), and "Swaziland has two common types of substance abuses and are regarded as socially acceptable: alcohol and marijuana..."(Youth Policy Press, 2015, p. 67). Swaziland was the former name of the present-day sub-Saharan African country, Eswatini (or the Kingdom of eSwatini) - Eswatini was adopted to replace the old name, Swaziland, effective June 1, 2018.

Notably though, compared to the male subgroup, the confidence intervals around the odds ratios of marijuana use in the female subgroup were wide, which indicates limited statistical power. This evidence would benefit from future studies that examine further the gender difference in the association between marijuana use and suicidal behavior among school-going adolescents in Eswatini. Beyond these, the current evidence of marijuana use being associated with increased odds of suicidal behavior is consistent with key findings by earlier and recent primary studies and systematic reviews (Almansour \& Siziya, 2017; Borges \& Loera, 2010; Carvalho et al., 2019; Gobbi et al., 2019; Tetteh et al., 2020). Evidence suggests that marijuana use has adverse health outcomes among adolescents: it impairs memory and judgement, and complicates the course of loneliness and depression, thereby elevating the risk for suicidal behaviors (Borges \& Loera, 2010; Paruk \& Burns, 2016; Volkow et al., 2014). Urgent concerted efforts are needed to formulate and implement regulations and control measures and policies against the unfettered access and use of marijuana (and other illicit drugs) by young people in the Eswatini (Mkhatshwa \& Malambe, 2019).

The findings of the current study that intrusion of privacy by parents within the family context — was associated with increased odds of suicidal behavior and, in contrast, parental monitoring and parental understanding were associated with reduced odds of suicidal behavior across the overall sample, are consistent with recent evidence from Eswatini (Almansour \& Siziya, 2017) and other Southern sub-Saharan African countries, including Mozambique (Pengpid \& Peltzer, 2020b; Seidu et al., 2020) and South Africa (Shilubane et al., 2012). Life-course models to understanding human development have suggested that parent-adolescent connectedness, parental understanding, parental supervision and supportive monitoring, and having supportive social networks present as strong determinants of wellbeing and positive (mental) health outcomes in adolescents across contexts and cultures (Patton et al., 2016; Viner et al., 2012). Consistent with previous studies from other parts of sub-Saharan Africa (Asante et al., 2017; Koyanagi, et al., 2019a, 2019b; Quarshie et al., 2020a; Seidu et al., 2020; Shayo \& Lawala, 2019), the current study has shown that food deprivation 
is associated with increased odds of suicidal behavior among school-going adolescents. Evidence suggests that young people who experience hunger tend to be irritable with high emotional responsiveness and may experience increased distraction that potentially could result in suicidal behaviors (Asante et al., 2017; Koyanagi, et al., 2019a, 2019b).

Adverse interpersonal factors and school-related problems (i.e., bullying victimization, physical fights, and truancy) showed strong associations with increased odds of suicidal behaviors in the current study; however, social support at school was associated with reduced odds of suicidal behaviors, particularly suicidal planning among males. These findings lend support to previous studies based on the WHO-GSHS data from other African countries that suggest that social adversities and adverse school-related experiences could lead to internalizing problems - including loneliness, depression, self-hate, feelings of guilt and shame - which in turn contribute to elevated risk for self-harm and suicidal tendencies among young people (Epstein et al., 2020; Koyanagi, et al., 2019a, 2019b; Page \& West, 2011; Pfledderer et al., 2019; Quarshie \& AndohArthur, 2020; Quarshie et al., 2015; Quarshie, 2020, 2020a, 2020b, 2020c; Siziya et al., 2007). Emerging qualitative findings from South Africa (Shilubane et al., 2012) and Ghana (Quarshie et al., 2020b, 2020c) suggest that peer social support, whether at school or within the community, contributes to effective coping, resilience, and recovery among adolescents experiencing emotional crises, thereby reducing the chances of suicidal behaviors. As recommended by a recent regional systematic review from sub-Saharan Africa (Quarshie et al. 2020c), future studies could consider exploring other social and interpersonal factors that promote mental health and "protect" school-going adolescents in Eswatini against the onset of suicidal behavior.

The key findings of the current study have implications for intervention and prevention efforts and policies. The multi-level and multi-contextual nature of the factors associated with suicidal behaviors among school-going adolescents underscores that need for multi-layered and multi-sectoral intervention and prevention programs and policy approaches. For example, while anti-bullying and anti-violence policies (Sivaraman et al., 2019) and inter-peer communication and interpersonal problem-solving programs are needed to enhance supportive school climate, social policies aimed at family poverty reduction, and supportive parenting programs are also required to improve the family environment. Schools should consider instituting help-seeking programs and mental health literacy events to improve help-seeking behaviors among students (at risk of) experiencing emotional crises, including anxiety and loneliness. Also, the evidence of the current study supports calls by the World Food Programme and other key stakeholders that the government of Eswatini must pay considerable attention to improving the Eswatini National School Feeding Programme (ENSFP) - in order to enhance the food and nutritional needs of school-going children and young people (Gandure et al., 2019). Recent evidence from Ghana suggests that school feeding programs improve educational and health outcomes among school-going young people (Awojobi, 2019). 


\section{Strengths and limitations of the study}

This secondary analysis represents one of the primary efforts to use a nationally representative dataset from Eswatini to examine the prevalence and correlates of suicidal ideation, planning, and attempt among school-going adolescents in an integrated, country-specific way. However, it is imperative to cautiously adopt and interpret the evidence, as there are notable limitations to the study. Each of the outcome variables (suicidal ideation, planning, and attempt) was assessed with a single item on the questionnaire - and this might have led to imprecise categorization of the behaviors. There is evidence to suggest that single-item measures could result in misclassification and imprecise estimation of past suicidal behaviors (Hom et al., 2016; Millner et al., 2015). Thus, future studies should consider the use of contextsensitive multi-item standardized measures of adolescent suicidal behaviors. The cross-sectional design used does not readily allow for causal inference to be made: the sequence of the occurrence of the exposure and outcome variables cannot be (clearly) delineated, as they were measured at the same time; thus, the associations between the exposure and outcome variables reported should not be misconstrued to mean causation.

Notably many of the exposure variables in the WHO-GSHS were measured during the past 30 days, while the outcome variables (suicidal behaviors) were measured during the past 12 months. Even though the previous 30 days may still be part of the previous 12 months, it would have reinforced a stronger sense of equivalence if both exposure and outcome variables were measured within the same timeframe (e.g., the previous 12 months).

Another notable observation of the current study is the higher prevalence estimates of suicidal planning than suicidal ideation. Apart from being counterintuitive, this observation is inconsistent with the process model or pathway idea of suicide (Klonsky et al., 2016; Millner et al., 2017). The conventional idea is that where ideation and planning are conceptualized as distinct phenomena, the estimates of ideation are expected to be equal to or higher than suicidal planning. We suspect that the contrary observation in the current study may be pointing to the recent debate related to the definitional and conceptualization difficulty related to distinguishing suicidal ideation from planning (House et al., 2020). Besides apparent inference of terminology from the specific items on the questionnaire that measured suicidal ideation and planning, it is not clear from the source of the dataset (WHO, 2020) what specific definitions of ideation and planning were applied in the WHO-GSHS. Thus, is it possible that while some participants might have taken both concepts (ideation and planning) to mean the same thing, other participants might have seen each concept to be distinct. Future studies can avoid this by providing participants with clear, explicit definitions of the outcome variables of interest.

Considering that suicidal behaviors are stigmatized in Eswatini (Horter et al., 2019), it is also possible that some of the participants in the current study might have provided socially desirable and guarded answers to the survey questions about suicidal behaviors. Also, the survey included only students who attended school on the day of the study; absentees were excluded. Evidence suggests that students who are (at risk of) engaging in health risk behaviors and mental health problems are more 
likely to be truant (Bovet et al., 2006; Epstein et al., 2020). These challenges might have presented as sources of bias to our analysis. As observed recently, future WHOGSHS should assess the sexual and gender minority status (lesbian, gay, bisexual, transgender, queer, or questioning [LGBTQ +] orientation) of the participants. Some school-going adolescents in the sub-region who identify as LGBTQ + are showing worrying vulnerability to self-harm and suicidal behaviors (Quarshie, 2020, 2020a, 2020b, 2020c). Secondary school net enrolment in Eswatini is estimated to be $46.3 \%$ (Government of the Kingdom of Eswatini \& UNFPA, 2019), which implies that our findings may not necessarily apply to all adolescents in the country; future studies could consider including out-of-school adolescents in the country for a relatively less biased sample. Besides the need for longitudinal studies to increase the certainty in identifying key risk and protective factors of suicidal behaviors (Franklin et al., 2017), carefully designed qualitative studies can also help explore the shared and unique personal meanings of suicidal behaviors among school-going adolescents in Eswatini (White et al., 2016).

\section{Conclusions}

Approximately 1.7 in 10 participants reported suicidal ideation, 1 in 5 reported suicidal planning, and about 1.5 out of 10 participants attempted suicide during the previous 12 months. Although the prevalence estimates of suicidal behaviors among school-going adolescents in Eswatini are compared with estimates from other subSaharan African countries, the multi-layered nature of the factors associated with suicidal behaviors among school-going adolescents underscores the need for multicontextual and multi-sectoral intervention and prevention programs and policy approaches targeted at mitigating the onset of suicidal ideation and possible transition to suicidal planning, attempt, and potential death by suicide in this young population.

Supplementary Information The online version contains supplementary material available at https://doi. org/10.1007/s43076-021-00094-y.

Acknowledgements We thank the Eswatini Ministry of Education and Training and the World Health Organization (WHO) and its partners for making publicly and freely available the data from the 2013 Eswatini WHO Global School-based Student Health Survey. More imperatively, we thank all the schoolgoing adolescents who contributed data for this survey.

Author contribution ENBQ and PA conceived, designed, and organized the study. PA performed the statistical analysis of the data, and ENBQ, KPVG, SAL, and PLN contributed to the interpretation of the data. ENBQ and PA drafted the manuscript, and KPVG, SAL, and PLN critiqued the manuscript for important intellectual content. All authors read and approved the final version of the manuscript.

Data availability The datasets used and/or analyzed during the current study are freely available from the WHO website: https://www.who.int/ncds/surveillance/gshs/country/en/.

Code availability Not applicable.

Declarations 
Competing interests The authors declare no competing interests.

Ethics approval The Institutional Review Board of the Country Office of WHO in Eswatini, and the Eswatini Ministry of Education and Training approved the survey. Policies laid out regarding consent procedures for participation in surveys were followed, including detachment of identifier information.

Consent to participate Official written permissions were obtained from the Eswatini Ministry of Education and Training, and the selected schools. Participating students provided written informed consent, while parental written consent was obtained from parents of participants aged 17 and younger.

Consent for publication Not applicable.

Open Access This article is licensed under a Creative Commons Attribution 4.0 International License, which permits use, sharing, adaptation, distribution and reproduction in any medium or format, as long as you give appropriate credit to the original author(s) and the source, provide a link to the Creative Commons licence, and indicate if changes were made. The images or other third party material in this article are included in the article's Creative Commons licence, unless indicated otherwise in a credit line to the material. If material is not included in the article's Creative Commons licence and your intended use is not permitted by statutory regulation or exceeds the permitted use, you will need to obtain permission directly from the copyright holder. To view a copy of this licence, visit http://creativecommons.org/licen ses/by/4.0/.

\section{References}

Aggarwal, S., Patton, G., Reavley, N., Sreenivasan, S. A., \& Berk, M. (2017). Youth self-harm in low-and middle-income countries: Systematic review of the risk and protective factors. International Journal of Social Psychiatry, 63(4), 359-375. https://doi.org/10.1177/0020764017700175

Almansour, A. M., \& Siziya, S. (2017). Suicidal ideation and associated factors among school going adolescents in Swaziland. African Health Sciences, 17(4), 1172-1177. https://doi.org/10.4314/ahs. v17i4.26

Angold, A., Erkanli, A., Silberg, J., Eaves, L., \& Costello, E. J. (2002). Depression scale scores in 8-17-year-olds: Effects of age and gender. Journal of Child Psychology and Psychiatry, 43(8), 1052-1063. https://doi.org/10.1111/1469-7610.00232

Asante, K. O., Kugbey, N., Osafo, J., Quarshie, E.N.-B., \& Sarfo, J. O. (2017). The prevalence and correlates of suicidal behaviours (ideation, plan and attempt) among adolescents in senior high schools in Ghana. SSM-Population Health, 3, 427-434. https://doi.org/10.1016/j.ssmph.2017.05.005

Awojobi, O. N. (2019). A systematic review of the impact of Ghana's school feeding programme on educational and nutritional outcomes. Agro-Science: Journal of Tropical Agriculture, Food, Environment and Extension, 18(2), 42-50. https://doi.org/10.4314/as.v18i2.8

Babyak, M. A. (2004). What you see may not be what you get: A brief, nontechnical introduction to overfitting in regression-type models. Psychosomatic Medicine, 66(3), 411-421. https://doi.org/10.1097/ 01.psy.0000127692.23278.a9

Berhane, Y., Canavan, C. R., Darling, A. M., Sudfeld, C. R., Vuai, S., Adanu, R., Bärnighausen, T., Dessie, Y., Bukenya, J. N., \& Guwatudde, D. (2020). The age of opportunity: Prevalence of key risk factors among adolescents 10-19 years of age in nine communities in sub-Saharan Africa. Tropical Medicine \& International Health, 25(1), 15-32. https://doi.org/10.1111/tmi.13339

Biswas, T., Scott, J. G., Munir, K., Renzaho, A., Rawal, L. B., Baxter, J., \& Mamun, A. (2020). Global variation in the prevalence of suicidal ideation, anxiety and their correlates among adolescents: A population based study of 82 countries. EClinicalMedicine, 82(24). https://doi.org/10.1016/j.eclinm. 2020.100395

Borges, G., \& Loera, C. R. (2010). Alcohol and drug use in suicidal behaviour. Current Opinion in Psychiatry, 23(3), 195-204. https://doi.org/10.1097/YCO.0b013e3283386322 
Bovet, P., Viswanathan, B., Faeh, D., \& Warren, W. (2006). Comparison of smoking, drinking, and marijuana use between students present or absent on the day of a school-based survey. Journal of School Health, 76(4), 133-137. https://doi.org/10.1111/j.1746-1561.2006.00081.x

Campisi, S. C., Carducci, B., Akseer, N., Zasowski, C., Szatmari, P., \& Bhutta, Z. A. (2020). Suicidal behaviours among adolescents from 90 countries: A pooled analysis of the global school-based student health survey. BMC Public Health, 20(1), 1-11. https://doi.org/10.1186/s12889-020-09209-z

Carvalho, A. F., Stubbs, B., Vancampfort, D., Kloiber, S., Maes, M., Firth, J., Kurdyak, P. A., Stein, D. J., Rehm, J., \& Koyanagi, A. (2019). Cannabis use and suicide attempts among 86,254 adolescents aged 12-15 years from 21 low-and middle-income countries. European Psychiatry, 56, 8-13. https://doi.org/10.1016/j.eurpsy.2018.10.006

Centers for Disease Control and Prevention. (2019). The social-ecological model: A framework for prevention. https://www.cdc.gov/violenceprevention/publichealthissue/social-ecologicalmodel.html

Epstein, S., Roberts, E., Sedgwick, R., Polling, C., Finning, K., Ford, T., Dutta, R., \& Downs, J. (2020). School absenteeism as a risk factor for self-harm and suicidal ideation in children and adolescents: A systematic review and meta-analysis. European Child \& Adolescent Psychiatry, 29, 1175-1194. https://doi.org/10.1007/s00787-019-01327-3

Franklin, J. C., Ribeiro, J. D., Fox, K. R., Bentley, K. H., Kleiman, E. M., Huang, X., Musacchio, K. M., Jaroszewski, A. C., Chang, B. P., \& Nock, M. K. (2017). Risk factors for suicidal thoughts and behaviors: A meta-analysis of 50 years of research. Psychological Bulletin, 143(2), 187. https://doi. org/10.1037/bul0000084

Gandure, S., Sacolo, T., \& Silaula, S. (2019). Decentralized evaluation: Evaluation of National School Feeding Programme in Eswatini 2010-2018. Final evaluation report. Ministry of Education and Training, World Food Programme Eswatini Country Office.

Glenn, C. R., Kleiman, E. M., Kellerman, J., Pollak, O., Cha, C. B., Esposito, E. C., Porter, A. C., Wyman, P. A., \& Boatman, A. E. (2020). Annual research review: A meta-analytic review of worldwide suicide rates in adolescents. Journal of Child Psychology and Psychiatry, 61(3), 294-308. https://doi.org/10.1111/jcpp.13106

Gobbi, G., Atkin, T., Zytynski, T., Wang, S., Askari, S., Boruff, J., Ware, M., Marmorstein, N., Cipriani, A., \& Dendukuri, N. (2019). Association of cannabis use in adolescence and risk of depression, anxiety, and suicidality in young adulthood: A systematic review and meta-analysis. JAMA Psychiatry, 76(4), 426-434. https://doi.org/10.1001/jamapsychiatry.2018.4500

Government of the Kingdom of Eswatini, \& UNFPA. (2019). Government of the Kingdom of Eswatini/ UNFPA 6th country programme evaluation (2016-2020). UNFPA.

Harber, C. (2017). Schooling in Sub-Saharan Africa. Policy, practice and patterns. Palgrave Macmillan.

Hawton, K., Saunders, K. E., \& O'Connor, R. C. (2012). Self-harm and suicide in adolescents. The Lancet, 379(9834), 2373-2382. https://doi.org/10.1016/S0140-6736(12)60322-5

Hom, M. A., Joiner, T. E., Jr., \& Bernert, R. A. (2016). Limitations of a single-item assessment of suicide attempt history: Implications for standardized suicide risk assessment. Psychological Assessment, 28(8), 1026-1030. https://doi.org/10.1037/pas0000241

Horter, S., Bernays, S., Thabede, Z., Dlamini, V., Kerschberger, B., Pasipamire, M., Rusch, B., \& Wringe, A. (2019). "I don't want them to know": How stigma creates dilemmas for engagement with Treatall HIV care for people living with HIV in Eswatini. African Journal of AIDS Research, 18(1), 27-37. https://doi.org/10.2989/16085906.2018.1552163

House, A., Kapur, N., \& Knipe, D. (2020). Thinking about suicidal thinking. The Lancet Psychiatry, 7(11), 997-1000. https://doi.org/10.1016/S2215-0366(20)30263-7

James, S., Reddy, S. P., Ellahebokus, A., Sewpaul, R., \& Naidoo, P. (2017). The association between adolescent risk behaviours and feelings of sadness or hopelessness: A cross-sectional survey of south African secondary school learners. Psychology, Health \& Medicine, 22(7), 778-789. https://doi.org/ 10.1080/13548506.2017.1300669

Kabiru, C. (2015). Adolescent girls' well-being in resource-poor settings. UNICEF. https://www.unicefirc.org/article/1150-adolescent-girls-well-being-in-resource-poor-settings.html

Kabiru, C. W., Izugbara, C. O., \& Beguy, D. (2013). The health and wellbeing of young people in subSaharan Africa: An under-researched area? BMC International Health and Human Rights, 13(1), 11. https://doi.org/10.1186/1472-698x-13-11

Klonsky, E. D., May, A. M., \& Saffer, B. Y. (2016). Suicide, suicide attempts, and suicidal ideation. Annual Review of Clinical Psychology, 12, 307-330. https://doi.org/10.1146/annurev-clinp sy-021815-093204 
Koyanagi, A., Oh, H., Carvalho, A. F., Smith, L., Haro, J. M., Vancampfort, D., Stubbs, B., \& Devylder, J. E. (2019a). Bullying victimization and suicide attempt among adolescents aged 12-15 years from 48 countries. Journal of the American Academy of Child \& Adolescent Psychiatry, 58(9), 907-918. https://doi.org/10.1016/j.jaac.2018.10.018

Koyanagi, A., Stubbs, B., Oh, H., Veronese, N., Smith, L., Haro, J. M., \& Vancampfort, D. (2019b). Food insecurity (hunger) and suicide attempts among 179,771 adolescents attending school from 9 highincome, 31 middle-income, and 4 low-income countries: A cross-sectional study. Journal of Affective Disorders, 248, 91-98. https://doi.org/10.1016/j.jad.2019.01.033

Li, L., You, D., Ruan, T., Xu, S., Mi, D., Cai, T., \& Han, L. (2020). The prevalence of suicidal behaviors and their mental risk factors among young adolescents in 46 low-and middle-income countries. Journal of Affective Disorders(advance online publication). https://doi.org/10.1016/j.jad.2020.11. 050

Lim, K.-S., Wong, C. H., McIntyre, R. S., Wang, J., Zhang, Z., Tran, B. X., Tan, W., Ho, C. S., \& Ho, R. C. (2019). Global lifetime and 12-month prevalence of suicidal behavior, deliberate self-harm and non-suicidal self-injury in children and adolescents between 1989 and 2018: A meta-analysis. International Journal of Environmental Research and Public Health, 16(22), 4581. https://doi.org/ 10.3390/ijerph16224581

Liu, X., Huang, Y., \& Liu, Y. (2018). Prevalence, distribution, and associated factors of suicide attempts in young adolescents: School-based data from 40 low-income and middle-income countries. PLoS ONE, 13(12), e0207823. https://doi.org/10.1371/journal.pone.0207823

McKinnon, B., Gariépy, G., Sentenac, M., \& Elgar, F. J. (2016). Adolescent suicidal behaviours in 32 low-and middle-income countries. Bulletin of the World Health Organization, 94(5), 340. https:// doi.org/10.2471/BLT.15.163295

Millner, A. J., Lee, M. D., \& Nock, M. K. (2015). Single-item measurement of suicidal behaviors: Validity and consequences of misclassification. PLOS ONE, 10(10), e0141606. https://doi.org/10.1371/ journal.pone.0141606

Millner, A. J., Lee, M. D., \& Nock, M. K. (2017). Describing and measuring the pathway to suicide attempts: A preliminary study. Suicide and Life-Threatening Behavior, 47(3), 353-369. https://doi. org/10.1111/sltb. 12284

Ministry of Education and Training, \& UNICEF. (2018). A report on out-of-school children in Eswatini. Eswatini Ministry of Education and Training and UNICEF.

Ministry of Sports Culture and Youth Affairs. (2015). Swaziland state of the youth report 2015. Ministry of Sport Culture and Youth Affairs, UNFPA, UNICEF, \& Swaziland National Youth Council.

Mkhatshwa, T. P., \& Malambe, G. B. (2019). Oral traditions: A tool for understanding alcohol and drug addiction in Swaziland. In Y. Ndasauka \& G. M. Kayange (Eds.), Addiction in South and East Africa: Interdisciplinary approaches (pp. 93-104). Palgrave Macmillan.

Mortier, P., Cuijpers, P., Kiekens, G., Auerbach, R., Demyttenaere, K., Green, J., Kessler, R., Nock, M., \& Bruffaerts, R. (2018). The prevalence of suicidal thoughts and behaviours among college students: A meta-analysis. Psychological Medicine, 48(4), 554-565. https://doi.org/10.1017/S0033 291717002215

Muehlenkamp, J. J., Claes, L., Havertape, L., \& Plener, P. L. (2012). International prevalence of adolescent non-suicidal self-injury and deliberate self-harm. Child and Adolescent Psychiatry and Mental Health, 6(1), 10. https://doi.org/10.1186/1753-2000-6-10

Nock, M. K. (2012). Future directions for the study of suicide and self-injury. Journal of Clinical Child \& Adolescent Psychology, 41(2), 255-259. https://doi.org/10.1080/15374416.2012.652001

Page, R. M., \& West, J. H. (2011). Suicide ideation and psychosocial distress in sub-Saharan African youth. American Journal of Health Behavior, 35(2), 129-141. https://doi.org/10.5993/ajhb.35.2.1

Paruk, S., \& Burns, J. K. (2016). Cannabis and mental illness in adolescents: A review. South African Family Practice, 58(sup1), S18-S21. https://doi.org/10.1080/20786190.2014.978106

Patton, G. C., Sawyer, S. M., Santelli, J. S., Ross, D. A., Afifi, R., Allen, N. B., Arora, M., Azzopardi, P., Baldwin, W., \& Bonell, C. (2016). Our future: A Lancet commission on adolescent health and wellbeing. The Lancet, 387(10036), 2423-2478. https://doi.org/10.1016/S0140-6736(16)00579-1

Peltzer, K., \& Pengpid, S. (2017). Lifestyle and mental health among school-going adolescents in Namibia. Journal of Psychology in Africa, 27(1), 69-73. https://doi.org/10.1080/14330237.2016. 1268293

Pengpid, S., \& Peltzer, K. (2020a). The prevalence and correlates of suicidal ideation, plans and suicide attempts among 15-to 69-year-old persons in Eswatini. Behavioral Sciences, 10(11), 172. https:// doi.org/10.3390/bs10110172 
Pengpid, S., \& Peltzer, K. (2020b). Suicide attempt and associated factors among in-school adolescents in Mozambique. Journal of Psychology in Africa, 30(2), 130-134. https://doi.org/10.1080/14330237. 2020.1746569

Perkins, D. F., \& Hartless, G. (2002). An ecological risk-factor examination of suicide ideation and behavior of adolescents. Journal of Adolescent Research, 17(1), 3-26. https://doi.org/10.1177/ 0743558402171001

Petroni, S., Patel, V., \& Patton, G. (2015). Why is suicide the leading killer of older adolescent girls? The Lancet, 386(10008), 2031-2032. https://doi.org/10.1016/S0140-6736(15)01019-3

Pfledderer, C. D., Burns, R. D., \& Brusseau, T. A. (2019). School environment, physical activity, and sleep as predictors of suicidal ideation in adolescents: Evidence from a national survey. Journal of Adolescence, 74, 83-90. https://doi.org/10.1016/j.adolescence.2019.05.008

Quarshie, E. N.-B. (2020). Self-harm and suicidal behaviour among LGBTQ+ young people: an overlooked public mental health issue in Africa. International Journal of Social Psychiatry(advance online publication). https://doi.org/10.1177/0020764020962145

Quarshie, E. N.-B., \& Andoh-Arthur, J. (2020). Suicide attempts among 1,437 adolescents aged 12-17 years attending Junior High Schools in Ghana. Crisis(advance online publication). https:// doi.org/10.1027/0227-5910/a000746

Quarshie, E. N.-B., Onyeaka, H. K., \& Oppong Asante, K. (2020). Suicidal behaviours among adolescents in Liberia. BMC Psychiatry, 20(572). https://doi.org/10.1186/s12888-020-02985-3

Quarshie, E.N.-B., Osafo, J., Akotia, C. S., \& Peprah, J. (2015). Adolescent suicide in Ghana: A content analysis of media reports. International Journal of Qualitative Studies on Health and WellBeing, 10(1), 27682. https://doi.org/10.3402/qhw.v10.27682

Quarshie, E. N.-B., Waterman, M. G., \& House, A. O. (2020a). Adolescent self-harm in Ghana: a qualitative interview-based study of first-hand accounts. BMC Psychiatry, 20(275). https://doi. org/10.1186/s12888-020-02599-9

Quarshie, E. N.-B., Waterman, M. G., \& House, A. O. (2020b). Adolescents at risk of self-harm in Ghana: a qualitative interview study exploring the views and experiences of key adult informants. BMC Psychiatry, 20(310). https://doi.org/10.1186/s12888-020-02718-6

Quarshie, E.N.-B., Waterman, M. G., \& House, A. O. (2020c). Self-harm with suicidal and nonsuicidal intent in young people in sub-Saharan Africa: A systematic review. BMC Psychiatry, 20(234), 1-26. https://doi.org/10.1186/s12888-020-02587-z

Randall, J. R., Doku, D., Wilson, M. L., \& Peltzer, K. (2014). Suicidal behaviour and related risk factors among school-aged youth in the Republic of Benin. PLOS ONE, 9(2), e88233. https://doi. org/10.1371/journal.pone.0088233

Rescorla, L., Achenbach, T. M., Ivanova, M. Y., Dumenci, L., Almqvist, F., Bilenberg, N., Bird, H., Broberg, A., Dobrean, A., \& Döpfner, M. (2007). Epidemiological comparisons of problems and positive qualities reported by adolescents in 24 countries. Journal of Consulting and Clinical Psychology, 75(2), 351-358. https://doi.org/10.1037/0022-006X.75.2.351

Rudatsikira, E., Siziya, S., \& Muula, A. S. (2007). Suicidal ideation and associated factors among school-going adolescents in Harare, Zimbabwe. Journal of Psychology in Africa, 17(1-2), 93-97. https://doi.org/10.1080/14330237.2007.10820150

Seidu, A.-A., Amu, H., Dadzie, L. K., Amoah, A., Ahinkorah, B. O., Ameyaw, E. K., Acheampong, H. Y., \& Kissah-Korsah, K. (2020). Suicidal behaviours among in-school adolescents in Mozambique: Cross-sectional evidence of the prevalence and predictors using the Global School-Based Health Survey data. PLoS ONE, 15(7), e0236448. https://doi.org/10.1371/journal.pone.0236448

Shagle, S. C., \& Barber, B. K. (1995). A social-ecological analysis of adolescent suicidal ideation. American Journal of Orthopsychiatry, 65(1), 114-124. https://doi.org/10.1037/h0079591

Shaikh, M. A., Lloyd, J., Acquah, E., Celedonia, K. L., \& Wilson, M. L. (2016). Suicide attempts and behavioral correlates among a nationally representative sample of school-attending adolescents in the Republic of Malawi. BMC Public Health, 16(1), 843. https://doi.org/10.1186/ s12889-016-3509-8

Shayo, F. K., \& Lawala, P. S. (2019). Does food insecurity link to suicidal behaviors among in-school adolescents? Findings from the low-income country of sub-Saharan Africa. BMC Psychiatry, 19(1), 227. https://doi.org/10.1186/s12888-019-2212-6

Shilubane, H. N., Ruiter, R. A., Bos, A. E., van den Borne, B., James, S., \& Reddy, P. S. (2012). Psychosocial determinants of suicide attempts among black South African adolescents: A qualitative analysis. Journal of Youth Studies, 15(2), 177-189. https://doi.org/10.1080/13676261.2011. 634400 
Shilubane, H. N., Ruiter, R. A., van den Borne, B., Sewpaul, R., James, S., \& Reddy, P. S. (2013). Suicide and related health risk behaviours among school learners in South Africa: Results from the 2002 and 2008 national youth risk behaviour surveys. BMC Public Health, 13(1), 926. https:// doi.org/10.1186/1471-2458-13-926

Sivaraman, B., Nye, E., \& Bowes, L. (2019). School-based anti-bullying interventions for adolescents in low-and middle-income countries: A systematic review. Aggression and Violent Behavior, 45, 154-162. https://doi.org/10.1016/j.avb.2018.07.007

Siziya, S., Muula, A. S., \& Rudatsikira, E. (2007). Prevalence and correlates of truancy among adolescents in Swaziland: Findings from the Global School-Based Health Survey. Child and Adolescent Psychiatry and Mental Health, 1(1), 15. https://doi.org/10.1186/1753-2000-1-15

Sun, G.-W., Shook, T. L., \& Kay, G. L. (1996). Inappropriate use of bivariable analysis to screen risk factors for use in multivariable analysis. Journal of Clinical Epidemiology, 49(8), 907-916. https://doi. org/10.1016/0895-4356(96)00025-X

Swannell, S. V., Martin, G. E., Page, A., Hasking, P., \& St John, N. J. (2014). Prevalence of nonsuicidal self-injury in nonclinical samples: Systematic review, meta-analysis and meta-regression. Suicide and Life-Threatening Behavior, 44(3), 273-303. https://doi.org/10.1111/sltb.12070

Tetteh, J., Ekem-Ferguson, G., Swaray, S., Kugbey, N., Quarshie, E. N.-B., \& Yawson, A. (2020). Marijuana use and repeated attempted suicide among senior high school students in Ghana: Evidence from the WHO Global School-Based Student Health Survey, 2012. General psychiatry, 33(6). https://doi.org/10.1136/gpsych-2020-100311

Turecki, G., Brent, D. A., Gunnell, D., O’Connor, R. C., Oquendo, M. A., Pirkis, J., \& Stanley, B. H. (2019). Suicide and suicide risk. Nature Reviews Disease Primers, 5(1), 1-22. https://doi.org/10. 1038/s41572-019-0121-0

Uddin, R., Burton, N. W., Maple, M., Khan, S. R., \& Khan, A. (2019). Suicidal ideation, suicide planning, and suicide attempts among adolescents in 59 low-income and middle-income countries: A population-based study. The Lancet Child \& Adolescent Health, 3(4), 223-233. https://doi.org/10. $1016 / \mathrm{s} 2352-4642(18) 30403-6$

UNDP. (2020). Human Development Report 2020. The next frontier: Human development and the Anthropocene. UNDP.

Vandenbroucke, J. P., Von Elm, E., Altman, D. G., Gøtzsche, P. C., Mulrow, C. D., Pocock, S. J., Poole, C., Schlesselman, J. J., Egger, M., \& Initiative, S. (2007). Strengthening the Reporting of Observational Studies in Epidemiology (STROBE): Explanation and elaboration. PLoS Medicine, 4(10), e297. https://doi.org/10.1371/journal.pmed.0040297

Viner, R. M., Ozer, E. M., Denny, S., Marmot, M., Resnick, M., Fatusi, A., \& Currie, C. (2012). Adolescence and the social determinants of health. The Lancet, 379(9826), 1641-1652. https://doi.org/10. 1016/S0140-6736(12)60149-4

Volkow, N. D., Baler, R. D., Compton, W. M., \& Weiss, S. R. (2014). Adverse health effects of marijuana use. New England Journal of Medicine, 370(23), 2219-2227. https://doi.org/10.1056/NEJMra1402 309

White, J., Marsh, I., Kral, M. J., \& Morris, J. (Eds.). (2016). Critical suicidology: Transforming suicide research and prevention for the 21 st century UBC Press.

WHO. (2014). Preventing suicide: A global imperative. WHO.

WHO. (2019). Suicide: key facts. https://www.who.int/news-room/fact-sheets/detail/suicide

WHO. (2020). Global school-based student health survey (GSHS) implementation. https://www.who.int/ ncds/surveillance/gshs/country/en/

Wilson, M., Dunlavy, A., Viswanathan, B., \& Bovet, P. (2012). Suicidal expression among school-attending adolescents in a middle-income sub-Saharan country. International Journal of Environmental Research and Public Health, 9(11), 4122-4134. https://doi.org/10.3390/ijerph9114122

Youth Policy Press. (2015). Youth and public policy in Swaziland. Druckerei Lokay.

Zalsman, G., Hawton, K., Wasserman, D., van Heeringen, K., Arensman, E., Sarchiapone, M., Carli, V., Höschl, C., Barzilay, R., \& Balazs, J. (2016). Suicide prevention strategies revisited: 10-year systematic review. The Lancet Psychiatry, 3(7), 646-659. https://doi.org/10.1016/S2215-0366(16) 30030-X 


\section{Authors and Affiliations}

Emmanuel Nii-Boye Quarshie ${ }^{1,2}$ (D) . Prince Atorkey ${ }^{3,4}$. Karla Patricia Valdés García ${ }^{5}$. Samuel Afotey Lomotey ${ }^{6}$. Pascal Landindome Navelle ${ }^{7}$

Prince Atorkey

princeatorkey@gmail.com

Karla Patricia Valdés García

karlavaldes@uadec.edu.mx

Samuel Afotey Lomotey

lomoteysamuel100@gmail.com

Pascal Landindome Navelle

pascallandindomenavelle@gmail.com

1 School of Psychology, University of Leeds, Leeds LS2 9JT, UK

2 Department of Psychology, University of Ghana, Accra, Ghana

3 School of Medicine and Public Health, University of Newcastle, Callaghan, Australia

4 Centre for African Research, Engagement and Partnership, University of Newcastle, Callaghan, Australia

5 Faculty of Psychology, Universidad Autónoma de Coahuila, Saltillo, Mexico

6 Department of Pharmacy, Accra Psychiatric Hospital, Accra, Ghana

7 School of Health and Life Sciences, Teesside University, Middlesbrough, UK 